\title{
Prostitution de rue en France : splendeur et misère de la prévention du sida
}

\author{
Catherine DESCHAMPS ${ }^{1}$
}

\begin{abstract}
Résumé
La prostitution est perçue comme un des espaces majeurs de danger en ce qui concerne le sida. La prévention peine à y inscrire ses marques. Confronté aux réalités de terrain et aux quelques rares chiffres disponibles, ce sentiment d'impasse se révèle en partie injustifié. Ce n'est d'ailleurs pas forcément la prostitution elle-même qui conduit aux prises de risque, mais des pratiques ou des situations associées (toxicomanie notamment). Quant aux prostituées, dans leur grande majorité, elles encouragent l'utilisation de préservatifs, non seulement pour se prémunir du virus mais également pour mettre les clients à distance physique et symbolique. Les pires ennemis de la prévention dans la prostitution ne semblent pas celles qui vendent des services mais ceux qui les achètent : depuis le début des années 2000, les clients sollicitent de plus en plus souvent des rapports non protégés.
\end{abstract}

Mots clés : prostituées, clients, rue, sida, " souillure", toxicomanie, migration.

Depuis la ratification par la France, en 1960, de la Convention de Genève, les contrôles sanitaires obligatoires et les registres policiers de la "prostitution notoire» sont interdits (article 6). Ces mesures ont réduit les situations de contrainte pesant sur les personnes prostituées, mais ont également entravé le suivi des maladies sexuellement transmissibles dans la prostitution. Avec l'arrivée du sida, ce flou n'a pas été sans conséquence. II faut attendre la fin des années 1980 pour qu'une enquête d'envergure [2] soit lancée. Le retour du "péril vénérien " conduit alors à un bouleversement des encadrements associatifs de la prostitution. II modifie aussi la gamme des pratiques sexuelles, sanitaires et préventives des prostituées.

Une enquête ethnographique réalisée à Paris et en proche banlieue entre janvier 2002 et avril 2005 [1] permet de dresser un tableau parcellaire des mécanismes de prévention des femmes prostituées de rue et de leurs clients et, à rebours, de questionner certaines représentations du sida dans la prostitution. Des tournées de nuit ou de jour dans différents secteurs de la ville ou

\footnotetext{
Laboratoire d'anthropologie sociale (EHESS - Paris) et Université libre de Bruxelles. La recherche qui motive le présent article a été financée par Sidaction et a fait l'objet d'un ouvrage [1].
}

des bois alentours, avec trois associations franciliennes, ont fait côtoyer plus de 600 femmes, hommes ou transgenres prostitués, anciennement installés sur les trottoirs ou récemment arrivés, de nationalités multiples, parfois consommateurs de produits illicites. Pour aide-mémoire et afin de limiter les effets de sidération, des notes ont été prises discrètement in situ. Le détail des rencontres était en outre consigné par écrit et en différé à chaque retour de tournée. De même, étaient rapportées dans le journal de bord nombre de réunions autour de la prostitution, que celles-ci fassent intervenir des politiques, des membres du milieu associatif, des chercheurs etc, et une revue de la presse quotidienne a été réalisée de 1999 à 2005. Ces descriptions et recueils de données permettent d'esquisser des tendances et des pistes d'analyses : en dépit du nombre de personnes entendues et vues, il s'agit incontestablement d'un travail qualitatif, non exhaustif ; une part d'incertitude et d'inconnu demeure. La méthode inductive a été préférée. La dizaine d'entretiens semi-directifs conduits au cours de l'automne 2002 montre un écart fort entre le discours médiatisable, qui laisse traces sur le magnétophone du chercheur, et l'observation des gestes et des paroles non soutirées ; ce constat n'a rien d'étonnant s'agissant d'une population discriminée dont les activités frôlent régulièrement la clandestinité. Quelques entretiens informels, répétitifs et non enregistrés, ont cependant été menés avec une cinquantaine de personnes prostituées, soit sur leur lieu d'exercice, dans leurs espaces de vie ou dans des cafés. II a ainsi été possible d'échanger longuement avec des femmes installées depuis plusieurs années en île-de-France et sur les trottoirs, de nationalité française, algérienne, marocaine, camerounaise, albanaise, bulgare ou roumaine. D'autres ont été plus rétives aux « confidences » hors du cadre associatif (les jeunes africaines anglophones) ou peu disponibles, les toxicomanes, voire impossible à aborder, telles les "marcheuses ", ces femmes chinoises d'âge mûr les plus récemment arrivées dans la prostitution à Paris.

Cette ethnographie des trottoirs, dont on retiendra essentiellement les apports sur la question des femmes et du $\mathrm{VIH} / \mathrm{sida}$, constitue le fil conducteur de l'article et le cœur des deux dernières parties. Toutefois, parce que les représentations du sida dans la prostitution et l'état actuel de la prévention dans ce milieu sont le produit d'une histoire et d'un contexte, en poser préalablement quelques termes éclaire les enjeux contemporains. 


\section{La prostitution : risque indirect ou échec de la prévention}

Les représentations du sida dans la prostitution ont peu changé au cours des 20 dernières années. D'abord, les personnes prostituées auraient à leur charge exclusive la prévention du sida dans la prostitution ${ }^{2}$. Elles porteraient la culpabilité de la transmission, voire " souilleraient " des milieux apparemment innocents. Ensuite, il y aurait, parmi elles, un pourcentage de séropositives forcément élevé, hier comme aujourd'hui. En corollaire, à l'instar de la représentation des toxicomanes au début de l'épidémie, les personnes prostituées seraient immatures, inaptes à faire régresser les risques au sein de leur groupe. Mais la prostitution symbolise-t-elle réellement une faillite de la prévention ? Au-delà des peurs, dont l'existence caractérise les sujets polémiques, que nous apprennent les enquêtes de terrain ?

\section{Le temps des chiffres : vieux " groupes à risque " et variabilité des situations}

En 1989, paraissent les premières données françaises concernant les prostituées et le VIH [3] : sur 998 femmes atteintes du sida répertoriées par la Direction générale de la Santé, 29 déclarent s'être prostituées. Parmi celles-ci, deux modes principaux de transmission existent : l'injection massivement et, en second lieu, les relations sexuelles avec des "partenaires réguliers", des clients habitués, avec qui la vigilance connaît des failles, mais plus sûrement des hommes qui ne paient pas, proxénètes ou amants. En majorité, ce n'est donc pas dans l'exercice même de la prostitution que ces femmes ont été exposées aux risques.

Ces résultats incitent à mettre l'accent sur la dangerosité de la toxicomanie. Anne Coppel, Lydia Braggiotti et al [2], dans leur recherche commencée en 1989 qui compare la rue Saint-Denis, au cœur de Paris, aux boulevards des Maréchaux du Nord de la capitale, confirment les inquiétudes. Alors que les femmes héroïnomanes sont concentrées aux portes de la ville, plus du quart de celles qui se prostituent dans cette zone géographique se déclarent séropositives. Par comparaison, moins de $5 \%$ des prostituées de la rue Saint-Denis, lesquelles nient souvent consommer des produits illicites, affirment être contaminées par le virus. Il est vrai qu'il s'agit de déclarations et que le sujet du sida est délicat à aborder frontalement avec les prostituées, notamment celles qui se revendiquent " traditionnelles $»^{3}$. Vers la même époque, est lancée à Lyon une recherche comparable à celle entamée à Paris [4]. L'insistance porte moins sur les femmes héroïnomanes que sur la proportion non négligeable des hommes, travestis et transgenres, à se prostituer sur l'espace public. Là encore, la prostitution est associée à un groupe dit « à risque ", déterminé

\footnotetext{
2 Une seule exception existe en France : la campagne adressée aux clients sous forme d'affichage publicitaire sur les bus lillois en 2005

3 C'est-à-dire les femmes installées depuis plusieurs années dans la prostitution et qui affirment répondre à un * code professionnel * précis, code qui fait la part belle au maintien du secret.
}

indépendamment du travail sexuel : I'homosexualité masculine ou, plus globalement, le sexe entre " hommes de naissance " ${ }^{4}$.

Le présent article est dédié à la prostitution féminine et au sida. Quelques-unes des différences sexuées dans la prostitution ont toutefois des incidences : les femmes peuvent faire une "carrière $n^{5}$ longue sur les trottoirs, tandis que les hommes quittent généralement la rue au-delà de 35 ans, voire dès 30 ans. Les travestis et les transgenres se situent entre ces deux extrêmes, tant en termes de longévité que pour les discriminations qu'ils et elles subissent.

Au cours du terrain ethnographique, réalisé près de 15 ans après les enquêtes citées plus haut, c'est dans la population des femmes qui reconnaissent une consommation de produits illicites et dans celle des travestis et des transgenres que le nombre de personnes se déclarant séropositives (dans le premier groupe) ou dénoncées comme telles (dans le second) est le plus important. Ces " aveux " ou ces délations tendent à confirmer ce qu'Anne Serre ${ }^{6}$ ou Neil McKeganey [7] observaient déjà dix ans plus tôt, tant en France que dans le reste du monde : une grande disparité de situation existe. La question de la pertinence de s'intéresser à la prostitution dans son ensemble doit alors être posée.

\section{L'incertitude statistique : l'arrivée des migrantes, l'instabilité et l'opacité}

Sous l'effet conjugué des arrivées massives de jeunes étrangères et du développement de la substitution, les femmes usagères de drogue par voie intraveineuse qui se prostituent semblent aujourd'hui avoir régressé en nombre relatif et en nombre absolu sur les trottoirs, singulièrement en région parisienne ${ }^{7}$. Ce constat se fonde sur une comparaison de mes observations dans les années 2000 avec ce que voyait Anne Coppel en 1989; sur des discussions avec d'anciens travailleurs sociaux d'intermède (service exclusivement parisien de l'Amicale du nid, dont l'action, initialement dirigée vers les femmes toxicomanes, s'est élargi à l'ensemble des femmes, puis à l'ensemble des personnes prostituées de rue dans la deuxième partie des années 1990), et sur la lecture des rapports annuels d'activité de cette même association. En revanche, le nombre absolu des hommes de naissance paraît rester stable. En France, après l'hécatombe des années 1980 et 1990, où beaucoup de femmes prostituées et droguées mouraient du sida, peu d'enquêtes statistiques continuent d'évaluer les taux de séroprévalence dans la prostitution. Tout juste,

La catégorie * homme de naissance ", contestable puisqu'elle assemble des groupes aux pratiques et socio-identités disparates, réunit formellement les hommes, les travestis et les transgenres.

La notion de * carrière * est ici empruntée à Howard Becker [5]

Anne Serre [6] notait également à propos du Brésil que, là aussi, les travestis prostitués étaient plus souvent séropositifs que les hommes.

A Lille. oủ une étude de terrain complémentaire a été conduite au printemps 2003, le nombre des femmes usagères de drogues par voie intraveineuse reste important, en proportion et en nombre absolu. Ces différences selon les villes laissent supposer que les politiques de réduction des risques n'ont pas eu partout les mêmes effets ; elles sont par ailleurs caractéristiques de l'attrait moindre de certaines agglomèrations pour les prostituèes immigrées. 
lorsque des femmes se font connaître comme prostituées dans certains centres de dépistage, produit-on quelques chiffres non représentatifs de l'ensemble. Ainsi les statistiques du Moulin Joly, centre de soins dont les locaux ont longtemps fait face au Bus des femmes : en 2004, aucune femme d'Europe centrale, venue le plus souvent pour des questions d'obstétrique, ne s'est révélée séropositive. En revanche, 9 à $10 \%$ des femmes d'Afrique sub-saharienne francophones l'étaient et $7 \%$ des africaines anglophones.

Cette polarisation des données du Moulin joli sur les immigrées est révélatrice d'un double mécanisme. D'abord, elle témoigne du changement de profil des femmes prostituées de la plupart des grandes villes françaises dès la deuxième partie des années 1990. Les jeunes étrangères, d'Europe de l'Est, d'Europe centrale et d'Afrique anglophone, deviennent un élément incontournable du décor urbain, accélérant le renouvellement des générations dans la prostitution et perturbant les enjeux idéologiques et préventifs. Ensuite, l'absence, dans les statistiques, de femmes de nationalité française, algérienne ou marocaine caractérise un état d'esprit particulier des personnes installées de longue date sur les trottoirs. Pour elles, dans un contexte de concurrence délétère, il s'agit de se distinguer des nouvelles migrantes, caricaturées et souvent exclues de l'entre-soi. Pour se faire, il importe de donner une image "professionnelle ", c'est-à-dire celle d'une probité totale quant au respect des consignes de prévention du VIH, et d'une réputation non entachée par l'aveu de consommations de drogues illicites ${ }^{8}$. En matière de sida et de drogue, les anciennes alternent alors un discours de perfection et un refus de discours. Cette difficulté à laquelle est confronté l'enquêteur ne dit rien, en creux, d'une mauvaise adhésion à la prévention dans l'exercice de la prostitution. Elle est essentiellement l'effet à retardement des discriminations que le virus a fait peser sur les prostituées au début de l'épidémie. Mais les silences, les refus de parole ou les discours d'excellence sont volontiers interprétés par les observateurs comme l'indice que les prostituées sont de fieffées menteuses et que, souvent séropositives, elles contreviendraient aux règles de prévention. Ainsi, la protection que les prostituées croient s'assurer, par l'évitement ou par l'embellissement, se retourne contre elles en termes de production de représentations durables.

À cet effet pervers de la résistance des " traditionnelles " à leur qualification comme "groupe à risque" s'ajoutent, pour alimenter les prévisions les plus pessimistes, la tradition d'opacité qui entoure les pratiques illégales, ainsi que les changements constants et rapides qui caractérisent le monde de la prostitution. L'arrivée, dans les métropoles occidentales, des jeunes étrangères déjà citées et, plus récemment, de femmes chinoises

\footnotetext{
8. Une * traditionnelle $\approx$ m'a raconté qu'il lui arrivait de faire des tests de dépistage du VIH, suite à des rapports non protégés avec des partenaires privés. Elle se rendait alors dans un centre de dépistage anonyme et gratuit situé à la fois loin de son lieu de racolage et loin de son domicile personnel. Ses collègues, à ses dires coutumières de la même pratique, n'en étaient informées sous aucun prétexte.
}

d'âge mûr, figure le rebondissement le plus contemporain. Elle ravive par ailleurs la peur des mafias, d'autant plus diabolisées que celles-ci viennent d'ailleurs.

En même temps que ces bouleversements s'opèrent sur les trottoirs français, l'épidémiologie du sida met en évidence l'exposition au risque des immigrés. Or les nouvelles recrues du pavé viennent de zones géopolitiques envers lesquelles s'exprime une suspicion, réactivant une vieille méfiance vis-à-vis des pays d'Afrique, et faisant surgir de nouveaux doutes concernant les pays de l'ancien bloc soviétique et la Chine. Toxicomanie et homosexualité masculine hier, migration aujourd'hui, la prostitution n'en finit pas d'être associée à des facteurs potentiels de risque.

\section{Les changements idéologiques : affrontement des arguments d'autorité}

Les premières enquêtes sur le travail du sexe et le sida d'abord, sur le changement du profil biographique de celles qui battent le pavé ensuite, ont provoqué deux revirements successifs dans l'appréhension idéologique de la prostitution en France. Avant les recherches du tournant des années 1980/1990, les associations, souvent catholiques, qui s'occupaient des prostituées étaient, pour l'écrasante majorité d'entre elles, exclusivement préoccupées par la prévention de la prostitution et la réinsertion des prostituées. Suite aux enquêtes précitées, deux nouvelles associations, dites de "santé communautaire ", ont été créées: le Bus des femmes à Paris, et Cabiria à Lyon. La logique de leur création ne se fonde plus sur la prévention de la prostitution, mais sur la prévention des risques dans la prostitution, tout particulièrement ceux imputables au sida. Au début des années 1990, le monopole de l'encadrement de la prostitution est donc tombé. Auparavant, le désir de rendre de la dignité faisait foi. II s'est ensuite trouvé en rivalité avec la volonté d'éviter la mort. Dans un cas comme dans l'autre, des arguments d'autorité sont mobilisés. Avant l'arrivée des immigrées sur les trottoirs, la réduction des risques sanitaires l'emporte d'une courte tête devant la lutte contre l'esclavage que représenterait la prostitution.

Mais, à la toute fin du xxe siècle, les étrangères ne peuvent plus passer inaperçues dans les grandes villes ${ }^{9}$. Leur visibilité est l'occasion d'un second revirement idéologique et associatif. Les jeunes associations de santé communautaire sont déboussolées : alors qu'elles pensaient pouvoir travailler à l'obtention de droits pour les " traditionnelles $" 10$, elles sont confrontées au retour manifeste des situations de contrainte, comme en témoignent

\footnotetext{
9 Leur présence et les dangers qu'elles encourent se trouvent largement médiatisés après l'assassinat dans le $19^{e}$ arrondissement, fin 1999, d'une jeune femme bulgare, Ginka Trifonova.

10 Les associations de santé communautaires adressées aux personnes prostituées sont nées du sida et en ont tiré des subsides. II faut toutefois reconnaitre que, dans les premières années de leur vie, les nouvelles associations ont plus timidement proposé des mesures originales de prévention du virus qu'elles n'ont réflèchi aux statuts professionnel et juridique les plus susceptibles de contenter les prostituées.
} 
des entretiens avec les acteurs et actrices de prévention qui ont vécu la transition. Puis les mouvements politiques s'emparent de la question. Le proxénétisme qui, ostensiblement, s'exerce sur beaucoup d'immigrées, justifie que l'urgence ne soit plus la réduction des risques, mais la disparition de la prostitution. La notion juridique de " respect de la dignité humaine ", qui gagne une place croissante dans le droit national et international depuis la Shoa, est convoquée, opposée à celle de "consentement ». Après la courte période où une appréhension pragmatique de la prostitution l'a emporté, l'ère d'un projet de société égalitaire ou libertaire (selon des tendances antagonistes qui se sont largement exprimées dans les journaux) gagne. Dans un contexte d'exacerbation des polémiques, le VIH est confiné à l'accessoire. D'autant que certains partisans du « respect de la dignité humaine " considèrent que toute mesure de prévention du sida incite à la prostitution. Entre dogmes et loi répressive ${ }^{11}$, il faut des conditions particulières pour que les femmes prostituées résistent à la baisse de vigilance.

\section{Éloignement de la " souillure »12 et protection contre le VIH}

Or, pour la plupart des prostituées rencontrées au cours du terrain, le préservatif ne protège pas seulement du mal vénérien. D'où le besoin d'un respect important des consignes de prévention de la part de ces femmes: pour garantir leur santé, mais aussi parce que le sens de la prostitution n'est pas le même pour celles qui vendent un service sexuel et pour ceux qui l'achètent.

\section{De la toilette aux capotes : une continuité de sens}

Au début des années 2000 , la préoccupation d'une toilette ponctuant chaque nouvelle passe a régressé : aucune de mes interlocutrices ne m'en a fait part spontanément, et seules celles qui disposent d'un studio évoquent parfois la toilette du client $^{13}$. Non que la toilette ne continue d'intervenir en fin de journée ou de nuit, de même qu'un changement de vêtement intervient entre la rue et le domicile privé. Mais ces "deux toilettes " séparent alors l'activité rémunératrice du reste de la vie, sans nécessiter une répétition quotidienne. Certes, les propos de nombreuses femmes évoquent un certain acharnement hygiéniste de fin de labeur. La valeur symbolique d'effacement de la souillure demeure à l'esprit. Pourtant, un objet semble avoir remplacé l'ancienne toilette répétitive : le préservatif. II intervient lors de chaque service sexuel comme auparavant les lavages. II s'inter-

\footnotetext{
11 La Loi pour la sécurité intérieure, du 18 mars 2003, fait du racolage public un délit passible d'une peine de 2 mois d'emprisonnement et d'une amende de 3750 euros. Des recherches, ailleurs qu'en France, montrent depuis longtemps combien les mesures répressives, politiques ou policières, fragilisent les prostituées face au sida [8].

12 La notion de « souillure * est empruntée ici à Mary Douglas [9].

13 Des discussions avec Anne Coppel et avec des femmes qui se prostituent depuis plus de vingt ans confirment ce changement radical survenu entre la période précédent le sida et la période actuelle.
}

cale également entre chaque corps. En plus de l'ancien rôle de ponctuation attribué à la toilette, la capote devient un tampon qui éloigne le sentiment de souillure. Daniel Welzer-Lang et al soupçonnaient déjà ces changements au début des années 1990 à Lyon [4] : "Avant l'utilisation du préservatif, l'imposition de mesures d'hygiène permettait de gérer leurs réactions vis-à-vis des odeurs. Elles lavaient les clients, avant et après le rapport, se lavaient de la même façon. »Certaines femmes rencontrées sur les trottoirs ont beau dire considérer la vente de services sexuels comme un commerce banal, de leurs déclarations à leurs gestes se dessine toute la complexité des tiraillements: ainsi, Iorsque des fellations sont faites sur un même client par deux prostituées, elles peuvent superposer les préservatifs sur le sexe de l'homme'14.

\section{Le préservatif : condition ou accompagnement de changements dans la prostitution}

Le remplacement des ablutions par les capotes, déplaçant la façon de réduire la contamination morale, répond également à d'autres modifications dans la prostitution de rue. Le nombre des personnes prostituées à disposer d'un local, un appartement ou un studio est en diminution, tandis qu'augmente en proportion le nombre de celles qui trouvent des abris de fortune pour exercer, en plein air, dans des espaces semi-publics ou des véhicules de clients. Les femmes d'Europe de l'Est et d'Afrique anglophone ne disposent souvent pas d'un espace privé où pratiquer leur activité. Précédemment, le développement de la prostitution en camionnette esquissait une tendance analogue. Or les différents lieux qui remplacent les studios ne disposent pas d'eau courante. Par défaut cette fois, le préservatif devient le produit de substitution de la toilette. Nombre des services sexuels ayant lieu dans des endroits accessibles au regard des collègues, ils peuvent devenir l'occasion d'un auto-contrôle : pour discréditer telle ou telle, on m'a parfois raconté l'avoir vue ne pas mettre de préservatif pour une fellation.

Par ailleurs, la diffusion des capotes, comme protection contre les maladies sexuellement transmissibles ou comme conjuration de la souillure, a modifié la gamme des services sexuels proposés. Ainsi, les personnes les plus récemment arrivées dans la prostitution ne proposent parfois pour seule prestation que des fellations. Certaines considèrent même avec dégoût d'autres pratiques, en particulier les pénétrations. À l'inverse, des femmes prostituées de longue date disent combien elles préfèrent " la baise à la pipe ", soit la pénétration vaginale. Pour ces dernières, la bouche reste associée à l'intime, moins évidemment monnayable ${ }^{15}$. De

\footnotetext{
Plusieurs femmes ou transgenres m'en ont fait part, dont je ne connais pas toujours le statut sérologique. Mais parmi elles, deux qui pratiquent ensemble déclarent être séronégatives, ne pas avoir l'hépatite $\mathrm{C}$ et savoir parfaitement que cette superposition n'aide pas à se protéger contre le sida.

Dans les productions pornographiques, alors que les pénétrations étaient des pratiques très couramment filmées il y a une vingtaine d'année, les fellations paraissent aujourd'hui les avoir supplantees.
} 
fait, ce sont toujours de jeunes africaines ou de jeunes femmes d'Europe de l'Est qui se plaignent lorsque les capotes distribuées par les associations n'ont pas bon goût. Ce souci des caractéristiques gustatives, signe que les préservatifs sont utilisés pour les fellations, n'a pas été rencontré de la part des traditionnelles qui fréquentent les structures ambulatoires.

Alors que beaucoup des anciennes ont connu la période pré-sida, les plus jeunes (les Africaines notamment) ont grandi avec ou, du moins, lorsqu'elles viennent de pays où les cas de séropositivité sont minimisés, elles ont commencé à se prostituer en France avec l'épidémie sans cesse répétée. Non sans cynisme, une Bulgare, parlant de son arrivée sur les trottoirs parisiens, l'évoque : "Quand je suis arrivée ici, j'ai découvert en même temps les trottoirs et les capotes. Avant de me balancer dans une rue, mon mac de l'époque m'a pris mon passeport en même temps qu'il me mettait dans les mains une boîte de préservatifs. C'était son cadeau de bienvenue. "Sans prétendre que les souteneurs se soucient systématiquement de la prévention des maladies sexuellement transmissibles - mais il faut bien qu'ils conservent leur source de revenus -, la distribution de préservatifs est parfois la seule forme de communication qu'ils tolèrent entre les travailleurs sociaux et les femmes qu'ils contrôlent.

Dans un autre registre, une jeune femme nigériane se fait la porteparole de son groupe face aux inquiétudes des acteurs de prévention. La discussion qui suscite sa réaction porte sur le sida et, plus généralement, la maladie. De jeunes concitoyennes présentes à ses côtés affirment unanimement que Dieu décide et que Dieu seul peut guérir, non les médicaments ou les traitements. La question qui brûle les lèvres finit par être posée par un éducateur spécialisé : " Mais alors, si Dieu est seul maître des destinées, à quoi bon utiliser des préservatifs ?» La réponse de la téméraire fuse : « Bien sûr qu'on met des capotes. Dieu peut guérir du sida, mais il ne faut pas fatiguer Dieu. " Beaucoup des femmes d'Afrique anglophone croisées sur les trottoirs accordent une grande importance à la religion. L'observation conduite sur le terrain de la prostitution féminine paraît contredire la représentation qui voudrait que la croyance rime mal avec la prévention du sida.

\section{Des outils de prévention sur le territoire : santé, propreté, morale}

Avant l'épidémie, les prostituées pouvaient aller vers les associations. Ce sont aujourd'hui les associations qui vont à leur rencontre, prenant pour prétexte d'apporter "à domicile " des protections. Des anciennes estiment que la distribution des préservatifs par les structures ambulatoires a rendu plus passives leurs jeunes collègues ${ }^{16}$. Selon cette interprétation, en même temps que le sida déplaçait la question de la prévention de la prostitution vers la préservation de la santé, il aurait instauré une

\footnotetext{
16 Les associations se dotent d'antennes mobiles essentiellement après la création des associations de santé communautaire. Le nom de la première d'entre elles en témoigne : le Bus des femmes.
}

forme plus pernicieuse de contrôle. Signe d'une rupture générationnelle, ce sont les femmes récemment arrivées en France qui sont les plus nombreuses à demander des préservatifs lors du passage des bus. Les Africaines anglophones peuvent même se chamailler pour en obtenir le plus possible.

Mais en même temps que des " traditionnelles " se sont plaintes de la nouvelle mobilité des structures qui gravitent autour de la prostitution, elles ont concouru au développement d'un moyen d'accès clandestin aux outils de prévention. Car elles ont ajouté les préservatifs, le gel, plus rarement les fémidons, à la liste des produits à vendre par leurs " fournisseurs spécialisés ", proxénètes selon la loi française, qui se déplacent tout autant sur les territoires de prostitution que les associations assermentées. La gamme des produits proposés à l'achat par ces fournisseurs ou distribués gratuitement par les bus n'est pas anodine. Singulièrement, les lingettes pour les mains inondent le marché de l'hygiène des prostituées. En complément de préservatifs qui mettent à distance, ces tissus blancs, facilement transportables, se diffusent pour assainir les parties du corps qui demeurent au contact du sexe des clients. Avec le VIH, les mains peuvent en effet rester les seules parties de l'épiderme des prostituées en prise directe avec la peau de ceux qui les paient. Elles sont à la fois l'espace du maintien de la pollution - les lingettes sont là pour l'éloigner, voire pour blanchir " l'argent sale » - et parfois l'unique lieu de domiciliation du plaisir pour les prostituées : beaucoup évoquent en termes de jouissance le moment où les billets pénètrent entre leurs doigts, en paroles ( « je prends mon pied quand je tâte les billets ", " le seul moment que j'aime, c'est quand ils me fourrent les billets dans la main" etc), ou par gestes (par exemple, le froissement des doigts pour signifier à distance à une collègue qu'un client a été généreux, ou la tension de plusieurs doigts pour dire la somme reçue, grand sourire à l'appui). Là encore, la lutte contre la souillure symbolique voisine avec la préservation de la santé et de l'hygiène. Lilian Mathieu [10] écrit que les prostituées " ont fait preuve d'une attitude responsable [par rapport au sida] que ne laissaient pas anticiper les représentations misérabilistes les plus courantes, les définissant par exemple comme des victimes de troubles psychologiques marquées par la passivité et incapables de faire face aux contraintes pesant sur elles. "Que l'on juge les raisons bonnes ou mauvaises, les prostituées, en tout cas dans le cadre de la sexualité tarifée, ont des intérêts multiples, personnels et circonstanciels, à adhérer aux consignes de prévention.

\section{Les zones d'ombre de la prévention}

Dans un climat de durcissement de la concurrence et de répression politique, il n'est pas assuré que la propension des prostituées à utiliser des protections résiste face à la pression économique. Le tableau n'est pas en tout point aussi idyllique que le laissent supposer les développements précédents. 


\section{La sexualité hors prostitution des prostituées}

Au cours du terrain, des femmes ont fréquemment dit être enceintes et vouloir se renseigner sur les possibilités d'avortement ou de suivi de grossesse. Elles ont toujours dit que le père était leur petit ami, ou du moins un garçon qui n'était pas un de leurs clients. Le géniteur pouvait être le proxénète ou un ami du proxénète. Les déclarations de grossesses non désirées ont, à une exception près, toujours été faites par des femmes originaires de pays d'Europe de l'Est ou d'Afrique anglophone. Que les femmes de certains pays exhibent leur grossesse plus que d'autres et continuent de travailler jusqu'au terme de celle-ci laisse croire qu'elles sont plus nombreuses à être enceintes, alors qu'elles peuvent n'être que plus visibles. Par ailleurs, les femmes d'Europe de l'Est ou d'Afrique anglophone qui se prostituent sont en moyenne plus jeunes que les femmes de nationalité française ou d'un pays du Maghreb, ce qui peut expliquer une plus grande proportion de grossesses dans leurs rangs. Impossible, donc, de conclure à une moins bonne utilisation des préservatifs de la part des nouvelles arrivantes. Les différences culturelles dans la gestion de la contraception interviennent aussi : la pilule, très utilisée en France, peut entrer en opposition avec d'autres modèles. L'avortement semble une pratique courante dans les zones sous ancienne influence communiste. Au demeurant, qu'il y ait ou non utilisation de contraceptifs ne dit rien d'une différence de gestion du VIH dans le cadre des rapports sexuels amoureux ou récréatifs selon l'origine. Si la grossesse est un indice de non-recours au préservatif, son absence ne doit pas faire supposer une protection systématique : nombre de femmes, qu'elles soient libres ou victimes de réseaux, admettent utiliser peu de préservatifs dès qu'elles pensent qu'une relation est stable.

Pour les prostituées, le recours au préservatif devient donc moins systématique en dehors des pratiques sexuelles monétarisées, où son usage répond à des logiques sociales autres. Plus une relation est fondée sur un sentiment amoureux et sur la durée, moins l'utilisation des protections perdure : en somme, lorsqu'elles ne font pas payer la sexualité, et plus encore si elles projettent une histoire dans l'avenir, les prostituées deviennent des femmes banales; lors des discussions, force est de constater qu'elles cherchent alors moins à répondre à une image de perfection. Certains clients habituels peuvent se trouver à mi-chemin entre partenaire privé et acheteur impersonnel de prestations. Isabelle de Vincenzi [11] écrit à propos des « nouvelles » toxicomanes des rues que « L'augmentation du risque d'infection par le VIH chez les utilisatrices de crack serait essentiellement liée aux relations sexuelles ambiguës que ces femmes entretiennent avec leurs fournisseurs et leurs partenaires de "fumerie". En effet, ces partenaires paient la relation sexuelle en fournissant le crack et non de l'argent, ce qui leur confère un statut entre celui de client (rapports le plus souvent protégés) et celui de partenaires "privés" (rapports le plus souvent non protégés). " Au cours de l'enquête, vers la Porte de Clignancourt, j'ai plus d'une fois rencontré des femmes toxicomanes disant attendre un client en particulier, connu et unique.

\section{Les demandes des clients et leurs représentations}

Les territoires de confins (les Portes Nord, Est ou Ouest de Paris et les bois de Vincennes et de Seine-et-Marne), où les femmes en situation irrégulière ou consommatrices de drogues sont les plus nombreuses, coïncident avec les endroits où la dénonciation de clients qui ne veulent pas utiliser de préservatifs est la plus fréquente. Dans ces "zones de relégation ", outre la récurrence de l'accusation, sa permanence dans le temps est significative. En revanche, dans les quartiers intérieurs de prostitution traditionnelle, rue Joubert ou Avenue Foch par exemple, les femmes évoquent moins de sollicitations pour avoir des rapports sexuels sans préservatif et, parallèlement, une variabilité de ce type de demandes depuis les débuts du sida. Elles déclarent que, si depuis 2000 ou 2001, les clients expriment à nouveau des souhaits de sexualité non protégée, l'expression de ces désirs avait quasiment cessé au long des dix années précédentes. Ainsi, deux phénomènes se télescopent : d'un côté, quelle que soit l'époque, les clients semblent se permettre plus de libertés avec les prostituées qu'ils considèrent fragiles, donc avec celles qu'ils pensent susceptibles d'accepter leurs propositions; de l'autre, indépendamment des " grades " dans la prostitution, un relâchement de la prévention se fait jour depuis six ans de la part des clients (ils répondent en cela à la tendance en population générale). De plus, aux dires de prostituées questionnées plus longuement, certains clients exercent des chantages: profitant de la concurrence, voire ayant avantage à attiser les conflits, ceux-là déclareraient, en mentant volontairement, qu'unetelle accepte des rapports non protégés, soit pour faire baisser les tarifs en cas d'utilisation du préservatif, soit pour convaincre de l'abandonner. Les femmes les plus tenues par le besoin d'argent (ou de produits) ont moins de moyens de résistance.

Malgré tout, les prostituées, y compris celles jugées influençables, se présentent souvent comme les dernières croisées des pratiques sans risque ${ }^{17}$. Mais les femmes fournissent un travail à des fins rémunératrices, alors que les hommes achètent de la sexualité, recherchent une forme de plaisir et sont dans l'espace de leurs loisirs. Les préservatifs ne peuvent avoir le même sens pour les unes et pour les uns. Outils professionnels et moraux pour les premières, ils semblent perçus comme des entraves aux corps à corps par nombre des seconds. Selon les prostituées et les lieux, entre la moitié et le quart d'entre eux commencent par essayer de convaincre de ne pas utiliser de préservatifs ; la majorité paraît toutefois finir par les accepter. Mais la prostitution, à

\footnotetext{
Les femmes toxicomanes exclues, mais elles ont moins que les autres femmes qui se prostituent recours à un discours prédigéré sur la prévention dans la prostitution, et elles se déclarent aussi nettement plus facilement séropositives ou VHC+ lorsqu'elles le sont. $\AA$ l'inverse, les pratiques préventives non conformes des autres femmes viennent de dénonciations, de même que l'existence chez les transgenres de personnes séropositives.
} 
l'inverse du modèle conjugal contemporain issu de la libération sexuelle, offre aux hommes qui le souhaitent la possibilité de ne se soucier que de leur plaisir personnel. Parallèlement, les clients considèrent que la prostitution est incompatible avec la reproduction : ils n'ont pas à s'inquiéter de la survenue accidentelle ou volontaire de grossesse. Cette " contraception symbolique » figurée par la prostitution résonne en écho avec l'étrange sentiment d'immunité de certains vis-à-vis du sida... Quelles que soient les campagnes à mettre en place, elles ne peuvent plus ignorer le rôle déterminant des clients. Car, comme cela a été écrit dès 2002 [12] : "Si être client de prostituée "n'existe pas", car cela est socialement occulté, alors la possibilité de conscience des risques est annulée. ”

\section{Conclusion}

Lors de sa réalisation, l'enquête ethnographique sur laquelle s'appuie en grande partie cet article a exploré l'articulation entre espace public, sexualité et argent. Le sida et les femmes n'en ont pas toujours été le cœur. Aussi, des parts d'ombre subsistent et les observations mentionnées, parfois impressionnistes, suggèrent d'autres terrains à étudier, dans des groupes plus ciblés, et ce pour mieux éclairer le sujet.

Chaque prostituée se sert continuellement en préservatifs dans les bus associatiff ${ }^{18}$. Ce geste rituel donne le sentiment que la prévention est omniprésente sur les trottoirs. Les discussions sur le sida sont cependant rares, à moins d'être sollicitées, voire soutirées. Des lacunes apparaissent alors dans les informations que les personnes ont sur le virus, en particulier dans deux groupes : les femmes âgées anciennement installées, et les femmes d'Europe de l'Est récemment arrivées. Mais si les connaissances parcellaires des « vieilles traditionnelles " et des jeunes étrangères de l'ancien bloc soviétique surprennent, pourtant leurs pratiques préventives semblent majoritairement adaptées dans le cadre de l'échange de services sexuels contre de l'argent. En outre, les rares chiffres disponibles suggèrent que ce n'est pas parmi ces prostituées que se rencontrent le plus de personnes séropositives. En dépit d'un bon usage des préservatifs et de connaissances plus approfondies que la moyenne, les migrantes venues d'Afrique sub-saharienne doivent retenir l'attention. Leurs taux de séroprévalence sont, de fait, plus élevés et, pour ce que le terrain peut laisser supposer, elles sont parmi celles qui ont le plus grand nombre de clients différents. La moindre faille dans la prévention peut statistiquement être lourde de conséquences. Plus encore, la dépendance aux produits illicites demeure le principal vecteur indirect de transmission du virus dans la prostitution. Les femmes pratiquent moins souvent l'injection, toutefois certaines se considèrent toujours toxicomanes plutôt que prosti-

\footnotetext{
18 La majorité des personnes prostituées rencontre les dispositifs associatifs. Stéphanie Pryen [13] note que trois quarts des prostituées qu'elle a interrogées à Lille ont croisé l'association Aides dans les derniers trois mois.
}

tuées. Le lieu du risque est alors associé à la drogue. C'est contre ce risque qu'elles se prémunissent en priorité, non contre les dangers liés à la sexualité. ${ }^{19}$

Dans la prostitution, le préservatif n'intervient pas tant comme acte de prévention du sida que comme vecteur de séparation, comme neutralisation du geste prostitutionnel. Ainsi, son relatif succès n'est pas forcément le signe d'une responsabilisation, mais il est l'indice d'une protection personnelle aux dimensions imaginaires très présentes. À revers, cette vision du préservatif comme objet d'éloignement et refus d'implication est confirmée par la difficulté à l'utiliser dans les couples stables fondés sur un attachement affectif.

\section{Références bibliographiques}

1. Deschamps C. Le sexe et l'argent des trottoirs. Paris: Hachette littératures, 2006.

2. Coppel A, Braggiotti L et al. Recherche action: Prostitution et santé publique. Paris : Rapport final à la Direction générale de la santé et à l'Agence française de lutte contre le sida, 1990

3. Vincenzi de I. Sida et prostituées. Revue des données françaises. BEH 1989 ; $40: 165-166$.

4. Welzer-Lang D, Mathieu L, Barbosa 0. Prostitution, les uns, les unes et les autres. Paris : Métaillié, 1993.

5. Becker HS. Outsiders. Paris : Métaillié, 1985 [1963].

6. Serre A. Prostitution: succès et impasse de la prévention. Transcriptase, Journal du Sida, Anrs : Xp conférence internationale sur le sida de Vancouver 1996, $n^{\circ}$ spécial : 22.

7. McKeganey N. Prostitution and HIV: What do we know and where might research be targeted in the future ? AIDS $1994 ; 8: 1215-1226$.

8. Harcourt C, Philpot R. Female prostitutes, AIDS, Drugs and alcohol in New South Wale. In AIDS, Drugs and Prostitution, MA Plant, Ed. London : Routledge, 1993.

9. Douglas M. De la souillure. Essai sur les notions de pollution et de tabou. Paris : La Découverte, 1967, 2001.

10. Mathieu L. Prostitution et sida. Sociologie d'une épidémie et de sa prévention. Paris : L'Harmattan, 2000.

11. Vincenzi de I. Travail sexuel: facteurs de risque et prévention du risque. Transcriptase $1998 ; 64: 19-20$.

12. CABIRIA. Rapport de synthèse, action, université, recherche. Lyon: Dragon Lune, 2002.

13. Pryen S. Stigmate et métier. Une approche socio-anthropologique de la prostitution de rue à Lille. Rennes : Presses universitaires de Rennes, 1999.

\footnotetext{
19 Les toxicomanes demandent nettement moins de préservatifs que les autres lors du passage des bus. Plus invisible, une autre forme de pratique d'injection peut par ailleurs inquièter, la prise d'hormones par seringues. Ce sont des travestis et non des femmes qui sont concernés. Et la question se pose différemment : à la différence de leurs consœurs précédemment citées, les travestis assument généralement une identité de prostitués, tout en rejetant une identité de toxicomanes.
} 